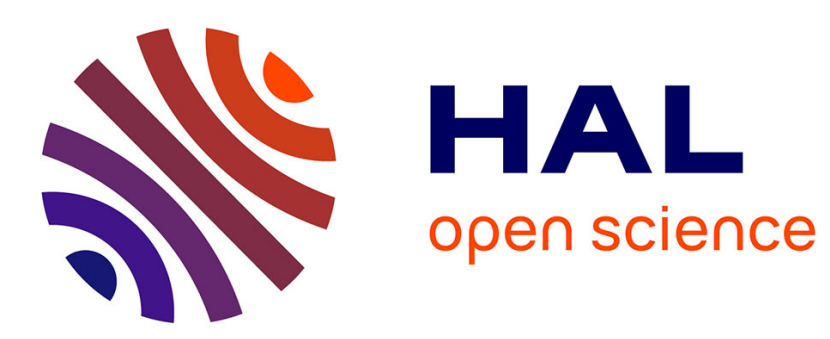

\title{
Growth histories and overlap distributions of diffusion-limited-aggregation clusters
}

\author{
Bernard Derrida, V. Hakim, J. Vannimenus
}

\section{To cite this version:}

Bernard Derrida, V. Hakim, J. Vannimenus. Growth histories and overlap distributions of diffusion-limited-aggregation clusters. Physical Review A, 1991, 43 (2), pp.888-899. 10.1103/PhysRevA.43.888 . hal-03282955

\section{HAL Id: hal-03282955 \\ https://hal.science/hal-03282955}

Submitted on 19 Jul 2021

HAL is a multi-disciplinary open access archive for the deposit and dissemination of scientific research documents, whether they are published or not. The documents may come from teaching and research institutions in France or abroad, or from public or private research centers.
L'archive ouverte pluridisciplinaire HAL, est destinée au dépôt et à la diffusion de documents scientifiques de niveau recherche, publiés ou non, émanant des établissements d'enseignement et de recherche français ou étrangers, des laboratoires publics ou privés. 


\title{
Growth histories and overlap distributions of diffusion-limited-aggregation clusters
}

\author{
B. Derrida \\ Service de Physique Théorique, Commissariat à l'Energie Atomique, Centre d'Etudes Nucléaires de Saclay, \\ 91191 Gif-sur-Yvette, France \\ V. Hakim and J. Vannimenus \\ Laboratoire de Physique Statistique, Ecole Normale Supérieure, 75231 Paris CEDEX 05, France
}

(Received 9 July 1990)

\begin{abstract}
The large variability in the ramified structure of diffusion-limited-aggregation clusters suggests an analogy between their branches and the phase-space valleys of spin glasses. We define the overlap $q$ of two growth sites, and study numerically the overlap function $q(x)$ of two- and three-dimensional aggregates. For isotropic aggregates we find that the average overlap decreases as a power law when their mass $N$ increases, indicating that the number of branches increases with $N$, and that the overlap function and its fluctuations obey a scaling law. Analytical results are presented for the infinite-dimensional limit on the Cayley tree. For anisotropic aggregates with $b$-fold symmetry $(b=2,3$, and 4$)$, the average overlap obtained numerically has a finite limit for large $N$, and the overlap function is in very good agreement with analytical calculations on a simplified model.
\end{abstract}

\section{INTRODUCTION}

The diffusion-limited-aggregation (DLA) model introduced by Witten and Sander ${ }^{1}$ is one of the most studied growth models, ${ }^{2}$ because of its appealing simplicity and its relevance to various experimental situations. After nearly one decade no complete analytical theory is available, in spite of the efforts spent. ${ }^{3}$ Recently several approaches have been proposed which aim to analyze in more detail the ramified morphology of the aggregates: wavelet transforms, ${ }^{4}$ ramification analysis, ${ }^{5}$ directed recursive fractal models, ${ }^{6}$ and spatio-temporal correlations of active zones. ${ }^{7}$ The overall shape of DLA clusters grown in a restricted geometry has also been determined. ${ }^{8}$

However, a basic unanswered question concerns the shape of large clusters grown in a radial geometry, without anisotropy: Is the number of branches well defined asymptotically? Or does it fluctuate from sample to sample? Or yet does it increase to infinity? Inspection of DLA aggregates indicates a large variability of branch weights and structure; indeed, so much so that the apparent number of main branches may often depend on the observer and is not a precisely defined quantity. This suggests an analogy between the branches and the phasespace valleys of random systems such as spin glasses: the analog of a microscopic state of the spin system is an active growth site. We show how a natural notion of cluster distance and of "overlap" $q$ between two growth sites can be introduced. The quantity so defined gives a precise, quantitative meaning to the qualitative notion that "two sites belong to the same branch," for irregular objects like DLA clusters, and we explore some of its properties in the present article.

Numerically, we will see that the average overlap $\langle q\rangle$ decreases slowly as the cluster mass $N$ increases, indicat- ing that for two- and three-dimensional isotropic DLA the effective number of branches grows with $N$ rather than saturating to a finite value. The distribution of overlaps is observed to follow a scaling law, where the scaling function depends on dimensionality, but which contains no free parameter if the scaling variable is expressed in terms of $\langle q\rangle$. Its fluctuations also follow a scaling law in the same variable, suggesting that the relative sample-tosample fluctuations remain finite for large $N$.

By contrast, for two-dimensional (2D) clusters grown using an anisotropic rule, the average overlap converges to a finite limit and the scaled distribution of overlaps is quite different from the isotropic case, for large enough clusters. An analytic calculation, based on a simplified model $^{9,10}$ for anisotropic growth, gives predictions for the scaled distributions in very good agreement with the numerical results.

The paper is organized as follows. In Sec. II the overlap $q$ for two sites and the overlap function $q(x)$ of a cluster are defined and examples are given, together with a description of the algorithms used. A numerical study of $2 \mathrm{D}$ and $3 \mathrm{D}$ isotropic DLA is presented in Sec. III, and the results are analyzed in terms of a scaling form for $q(x)$ and its fluctuations. Comparison is made with analytical results for the Cayley-tree limit. Section IV is devoted to $2 \mathrm{D}$ anisotropic growth. It contains numerical results on clusters grown with uniaxial, threefold and fourfold symmetry, and analytical results for the general $p$-fold symmetry on a simplified model. The details of the analytical calculations on the Cayley tree are given in the Appendix.

\section{BASIC DEFINITIONS AND EXAMPLES}

\section{A. Growth histories and overlaps}

Let us consider an aggregate, all particles of which bear a tag indicating their rank of arrival: the first parti- 
cle (the seed or root) bears the number 1 ; the most recent one, the number $N$. In addition, one keeps track for every particle of its antecedent, i.e., the (unique) particle on which it collided with at the end of its diffuse motion, so the whole succession of "ancestors" between the root and a given growth site (its "history") can be reconstructed.

Now, let a pair of test particles $A$ and $B$ diffuse independently until they come into contact with the aggregate, and let $k$ be the rank of their closest common ancestor, i.e., the time when the histories of the two test particles bifurcated (see Fig. 1). We define the overlap $q_{A B}$ of the pair $(A, B)$ as

$$
q_{A B}=\frac{k}{N} .
$$

If the only common ancestor is the root, $q=1 / N$ and vanishes in the thermodynamic limit. In particular, for a perfectly symmetric cluster with $b$ identical branches, the probability that $q=0$ would be $(b-1) / b$ for $N \rightarrow \infty$. The other limit $q=1$ is reached only if the two test particles hit precisely the last particle added to the cluster. In fact, for large $N, q \cong 1$ when the two test particles land close to the tip of the same branch, as their latest common ancestor is then a recently added particle with a probability tending to 1 when $N \rightarrow \infty .^{11}$ Intermediate values of $q$ measure the kinship of the test particles, in the sense that they share a partially common history and belong to subbranches that split off from the same main branch.

If now many test pairs are launched the probability distribution $P(q)$ is obtained. The rule of letting the test particles diffuse before calculating $q$ ensures that more weight is given to the active growth zones near the tips than to the screened fjords. ${ }^{12}$

For one given aggregate the distribution $P(q)$ has a very irregular structure, which reflects the irregular shape of the cluster, and it is more convenient to study the integrated distribution

$$
x(q)=\int_{0}^{q} P\left(q^{\prime}\right) d q^{\prime},
$$

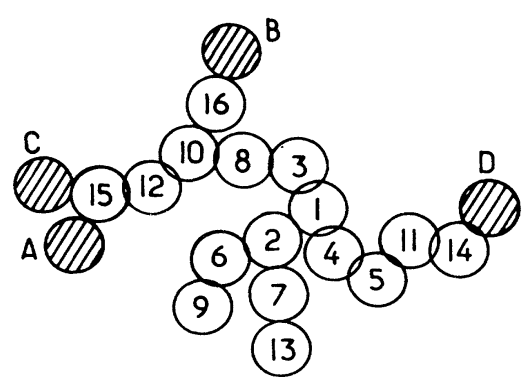

FIG. 1. An aggregate with its particles (open circles) numbered according to their order of arrival. The overlaps of the two pairs $(A B)$ and $(C D)$ of test particles (hatched circles) are, respectively, $q_{A B}=\frac{10}{16}$ and $q_{C D}=\frac{1}{16}$.

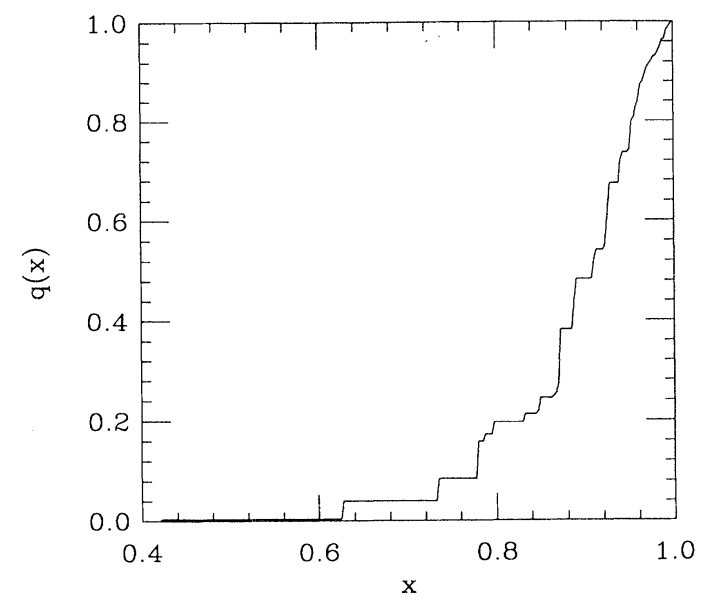

FIG. 2. Overlap function $q(x)$ of one aggregate (mass $N=500$ particles), computed using $10^{4}$ test pairs.

or, as customary for spin glasses, the inverse function $q(x)$, which gives the value of $q$ such that $q_{A B} \leq q$ for a fraction $x$ of the test pairs.

A particular realization of $q(x)$ for a 2D off-lattice aggregate is shown in Fig. 2. It displays many large steps that are not due to the finite number of test particles used but to the irregular branch structure of the aggregate. For each step, the value of $q$ indicates that a branch splitting occurred at "time" $t=N q$, and if the two subbranches created have, respectively, growth probabilities $p_{1}$ and $p_{2}$ at the end of the process, the step width is given by $2 p_{1} p_{2}$. The average of $q(x)$ over many samples yields a smooth curve, but by analogy with spin glasses it is possible and interesting to study the fluctuations in order to know if self-averaging takes place for large enough aggregates. ${ }^{13,14}$

Other definitions of the overlap can be considered, for instance, by making only reference to the spatial structure of the aggregate, irrespective of the temporal correlations (but keeping the diffusion-weighted average). For the DLA, we checked that this would not substantially change the results below, as the distance of a particle from the root is on average monotonically related to its time of arrival, and the present definition of overlaps is both simple and convenient for comparison with analytical calculations.

\section{B. Algorithms}

To generate isotropic 2D and 3D DLA clusters we used a simple off-lattice algorithm where initially a circular (spherical) particle of diameter $a$ is launched at a random position on a circle (sphere) of radius $R+\delta$ centered on the root particle, $R$ being the outer radius of the cluster and $\delta$ being a few times the particle diameter $(\delta=3 a$ was used, in general). Then, at every step the distance $d_{\text {min }}$ of the particle to the closest point of the cluster is determined: if $d_{\min }<a$, the diffusing particle stops and 

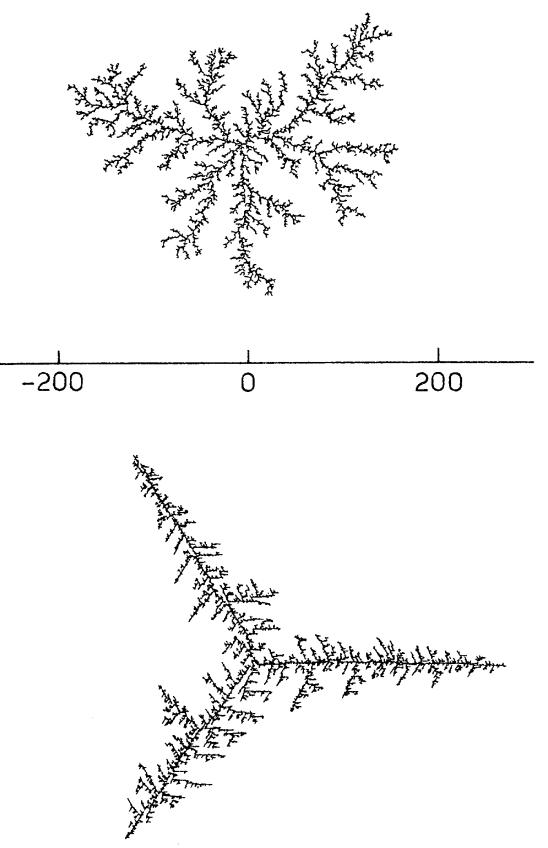

FIG. 3. Two aggregates of $10^{4}$ particles grown using the algorithms described in this paper: (upper panel) isotropic; (lower panel) with threefold anisotropy (no noise reduction).

becomes part of the cluster; its antecedent is the closest particle previously determined. Otherwise, it jumps to a random position on a circle of radius $\left(d_{\min }-\rho\right)$, with $\rho=0.8 a$. This algorithm produces DLA clusters of slightly overlapping particles. Following standard practice, the particle is lost if the distance to the root becomes too large ( $>4 R$, here). The calculations were run in FORTRAN on the Convex C-1 computers at Saclay and Ecole Normale Supérieure, using the standard random number generator of the computer. No specific algorithm improvements were implemented, in order to keep the programs as simple and versatile as possible, except for vectorization of the calculation of the distance $d_{\min }$ of the diffusive particle to the aggregate.

To grow anisotropic clusters we used the "antenna" method: ${ }^{15}$ the particle diffuses as previously, but when it comes into contact, it is rotated towards fixed directions depending on the contact sector. For the uniaxial case, the degree of anisotropy can be modulated by varying the ratio $\alpha$ of the angular sectors for accretion along the horizontal and vertical axes. For fourfold anisotropy we used the standard noise-reduction method ${ }^{16}$ to strengthen anisotropy effects: every antenna of a cluster bears a counter which is incremented each time the antenna is hit by a diffusing particle. Accretion takes place only if the counter reaches a threshold $m$, the counters on the other antennas of the site are not put back to zero after accretion, and for the test particles the sticking process is done without any noise reduction (Fig. 3).

\section{ISOTROPIC AGGREGATES}

\section{A. Average overlap}

In order to reduce statistical uncertainties and allow the precise study of size effects, we generated a rather large number ( $N_{s}=100$ or more) of middle-sized isotropic aggregates: mass $N \leq 4000$ for $d=2, N \leq 2400$ for $d=3$. For each sample the overlap distribution was obtained by launching a number $N_{t}$ of test particles, storing their growth history, then drawing at random a number $N_{p}$ of pairs from the list, among the $N_{t}^{2}$ possibilities. Typically, $N_{t}$ was equal to 1000 and $N_{p}$ to 2500 for the largest samples. We checked that this procedure introduced negligible bias, as compared with the use of independent pairs, while saving much computer time for large samples (at the expense of main memory storage), and that this value of $N_{p}$ was large enough to give $q(x)$ for each aggregate with sufficient accuracy. To obtain $q(x)$, the $N_{p}$ values of
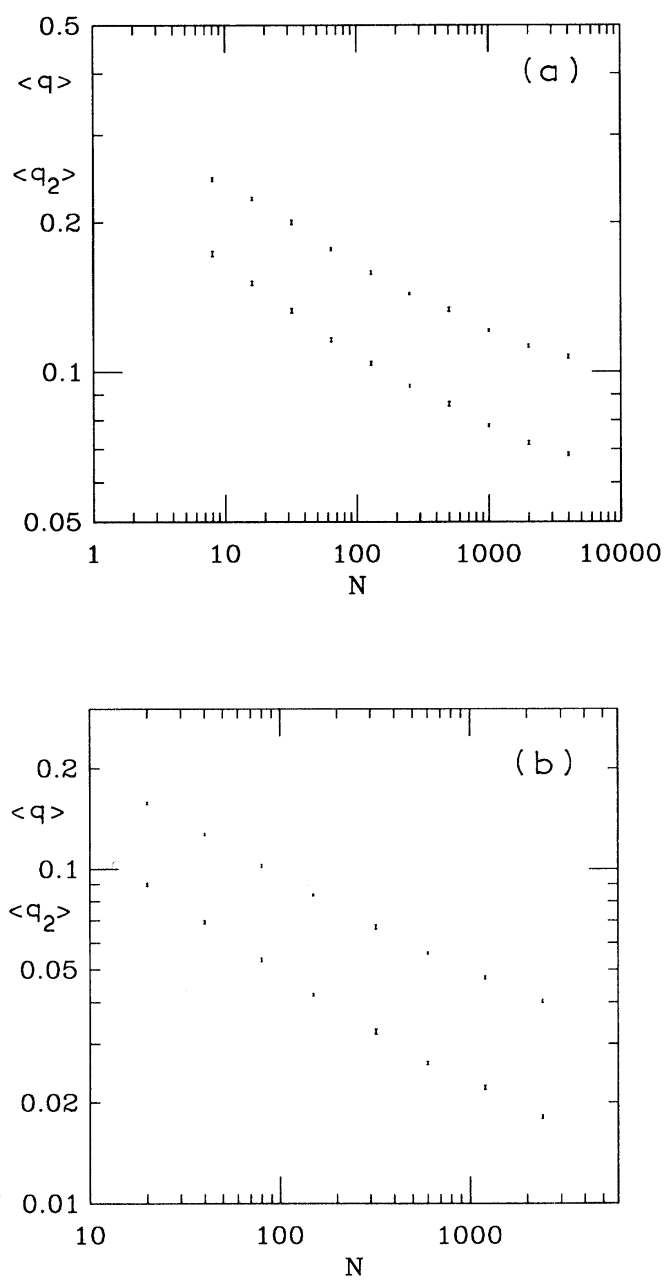

FIG. 4. Variation of the average overlap $\langle q\rangle$ and the average square overlap $\left\langle q_{2}\right\rangle$ with aggregate mass $N$ : (a) $d=2$; (b) $d=3$. The error bars correspond to one standard deviation. 
$q$ were stored, then sorted in increasing order, so that the $k$ th value gave $q\left(k / N_{p}\right)$. Finally, averages were taken at fixed $k$, over a number of samples.

For a given sample we measure the mean overlap $\bar{q}$ by

$$
\bar{q}=\frac{1}{N_{p}} \sum_{\text {pairs } A, B} q_{A B} .
$$

The average of $q$ over many samples, which we denote $\langle q\rangle$, was found to decrease with size (Fig. 4), without sign of saturating to a finite value. In $d=2$, it decreased from $\langle q\rangle=0.200$ for $N=32$ to 0.107 for $N=4000$. Analysis via a simple power-law dependence

$$
\langle q\rangle \approx C N^{-\beta}
$$

yields effective values of $\beta$ that decrease steadily from $\beta \approx 0.14$ in the range $N=500-1000$ to $\beta \approx 0.07$ in the range $N=2000-4000$. On the other hand, an expression of the form

$$
\langle q\rangle \approx C^{\prime} / \ln \left(N / N_{0}\right)
$$

gives a good fit in the range $N=32-2000$, as shown in Fig. 5.

Of course, one cannot exclude that $\langle q\rangle$ might converge to a finite but very small value. However, it will be shown later on that, for anisotropic star-shaped clusters with a fixed number $b$ of branches, the average overlap has a finite limit when the mass $N \rightarrow \infty$, of order $b^{-1}$ for large $b$. As there is no particular reason to expect that asymptotically isotropic DLA clusters have 13 branches, say, we tentatively conclude that $\langle q\rangle$ vanishes for large $N$, with a very small exponent $\beta$; or, more likely, with a logarithmic law, Eq. (4b). For very large off-lattice clusters, $N$ up to $10^{6}$, the apparent number of branches seems indeed to grow slowly. ${ }^{17}$

For $d=3$, a power-law fit is in better agreement with the data than a logarithmic decay. The measured value of the exponent $\beta$ decreases, however, a little with size, from

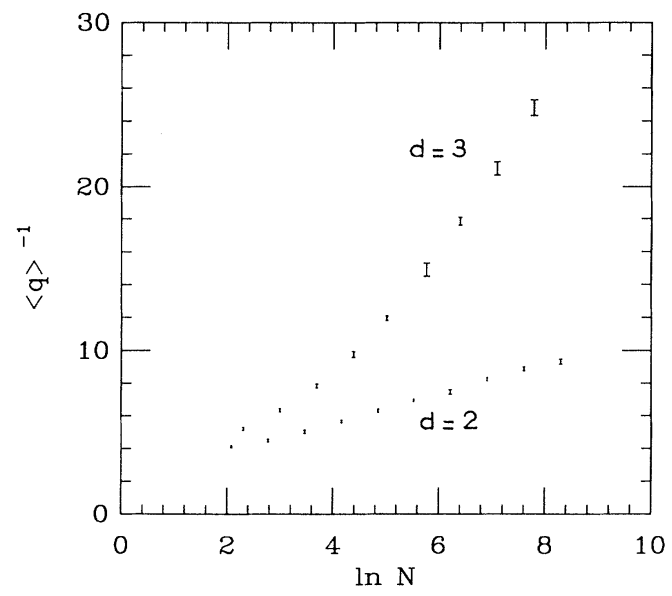

FIG. 5. Inverse of the average overlap $\langle q\rangle^{-1}$ vs $\ln N$, for $d=2$ and $d=3$. $\beta=0.29 \pm 0.01$ in the range $N=150-600$ to $\beta=0.24 \pm 0.02$ in the range $600-2400$.

\section{B. Scaling law for $q(x)$}

We also analyzed the mean square overlap $\overline{q^{2}}$ given for one aggregate by

$$
\overline{q^{2}}=\frac{1}{N_{p}} \sum_{\text {pairs } A, B} q_{A B}^{2}
$$

and its average over many samples, which we denote $\left\langle q_{2}\right\rangle$. From our numerical data we observed that $\left\langle q_{2}\right\rangle$ has very nearly the same dependence over $N$ as $\langle q\rangle$ (and not as $\langle q\rangle^{2}$ ): the ratio $\left\langle q_{2}\right\rangle /\langle q\rangle$ remains practically constant in two dimensions, and in $d=3$ it decreases much more slowly than each of the terms separately. The same remark holds for the quantity $\left\langle\overline{q^{2}} / \bar{q}\right\rangle$, that is, if one takes the average over many samples only after computing the ratio of the moments of $P(q)$ (see Fig. 6).

This suggests that the (averaged) distribution of overlaps $P(q)$ has a simple size dependence and can be decomposed into a contribution concentrated near $q=0$ and converging to a $\delta$ function for $N \rightarrow \infty$ plus a nontrivial part that depends on $N$ only via a multiplicative factor

$$
P(q) \cong(1-\epsilon) \delta(q)+\epsilon P^{*}(q),
$$

where $\epsilon \rightarrow 0$ for $N \rightarrow \infty$ and $P^{*}(q)$ is independent of $N$ and normalized: $\int_{0}^{1} P^{*}(q) d q=1$. A plausible interpretation of such a form for $P(q)$ is that the effective number of branches grows with $N$ like $\epsilon^{-1}$ and the average distribution may be viewed as just the superposition of contributions from individual branches. Then,

$$
\langle q\rangle=\int_{0}^{1} q P(q) d q \cong \epsilon \int_{0}^{1} q P^{*}(q) d q,
$$

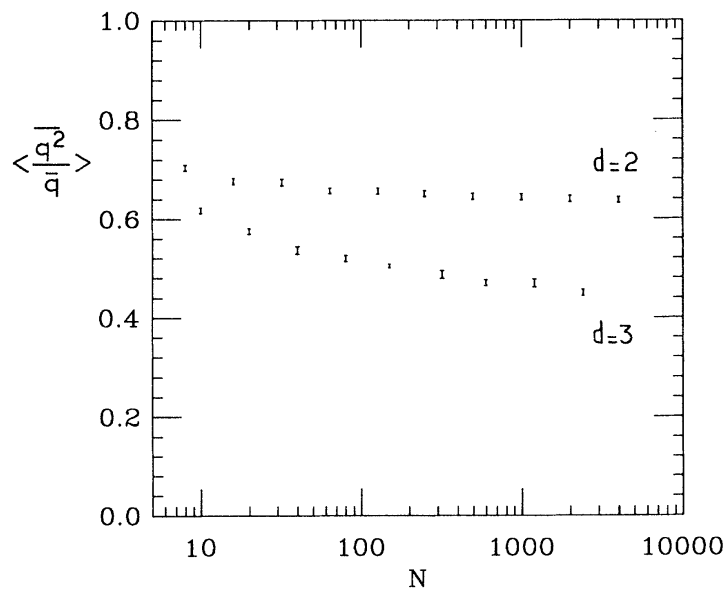

FIG. 6. Mass dependence of $\left\langle\overline{q^{2}} / \bar{q}\right\rangle$ [the average over samples is taken after computing the ratio of the first and second moments of the overlap distribution $P(q)]$. 


$$
\left\langle q_{2}\right\rangle=\int_{0}^{1} q^{2} P(q) d q \cong \epsilon \int_{0}^{1} q^{2} P^{*}(q) d q,
$$

and the ratio $\left\langle q_{2}\right\rangle /\langle q\rangle$ converges to a finite value even though $\langle q\rangle$ vanishes for large $N$. The integrated distribution $x(q)$ is given by

$$
x(q)=\int_{0}^{q} P\left(q^{\prime}\right) d q^{\prime} \cong 1-\epsilon+\epsilon \int_{0}^{q} P^{*}\left(q^{\prime}\right) d q^{\prime},
$$

so that

$$
1-x(q) \cong \epsilon \int_{q}^{1} P^{*}\left(q^{\prime}\right) d q^{\prime} .
$$

Eliminating $\epsilon$ in favor of a directly measured moment of $P(q)$, for instance $\langle q\rangle$, via Eq. (7) and inverting Eq. (10), one finally obtains a scaling expression with no adjustable parameter

$$
q(x) \cong F((1-x) /\langle q\rangle), \quad N \rightarrow \infty .
$$

It is easily shown by injecting relation (11) back into Eq. (7) that with this choice of variables the function $F(z)$ is normalized:

$$
\int_{0}^{\infty} F(z) d z=1 \text {. }
$$

The scaling function $F(z)$ depends on the space dimensionality and is a characteristic property of the growth process.

A plot of $q$ versus $z=(1-x) /\langle q\rangle$ for different sizes is presented in Fig. 7 for $d=2$. The results fall on a universal curve, showing that Eq. (11) holds at least in the range of sizes studied, where $\langle q\rangle$ itself varies by $40 \%$. The results for $d=3$ are displayed in Fig. 8 and also show good agreement with a scaling form.

The nontrivial part of the overlap distribution $P^{*}(q)$ can then be reconstructed from $F(z)$, if desired, through the relation $P^{*}(q) \sim|d F / d z|^{-1}$, with $q=F(z) . P^{*}(q)$ is a monotonically decreasing function of $q$ in $d=3$, as easily seen from Fig. 8. In two dimensions there is an inflection

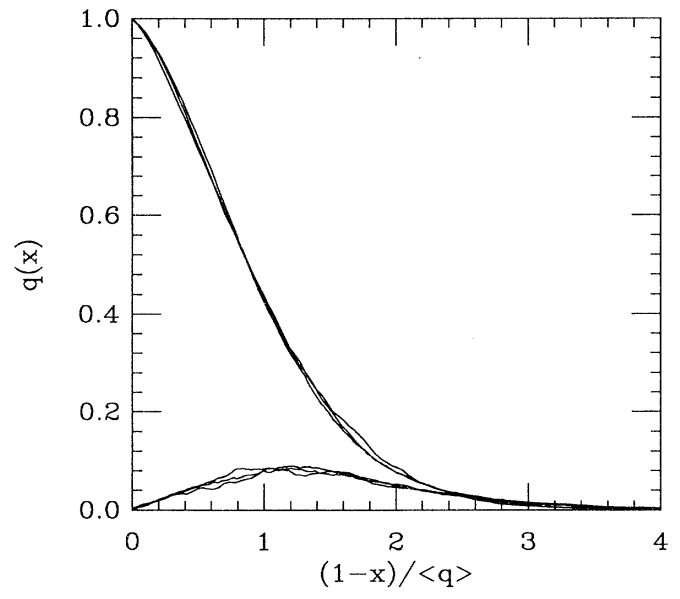

FIG. 7. Overlap function $q(x)$ and its fluctuations $\Delta q(x)$ (lower curves) vs the scaling variable $z=(1-x) /\langle q\rangle$, for twodimensional aggregates of mass $N=250$ (400 samples), 1000 (200 samples), and 4000 (100 samples).

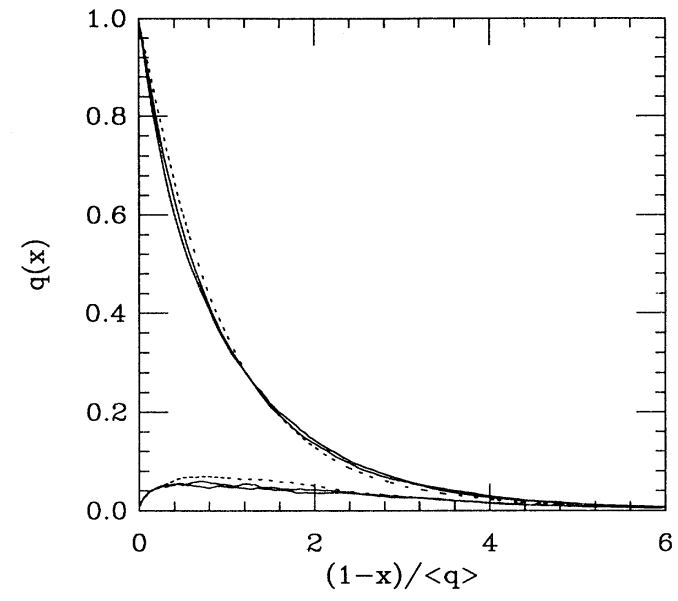

FIG. 8. Overlap function $q(x)$ and fluctuations $\Delta q(x)$ for three-dimensional aggregates of mass $N=150$ (dotted lines, 400 samples), 600 (200 samples), and 2400 (100 samples).

point in $F(z)$, which implies a minimum for $P^{*}(q)$, but its location moves closer to $q=1$ when $N$ increases, and our data are not extensive enough to rule out the possibility that the minimum of $P^{*}(q)$ is a size-dependent effect.

The quantity $q(x)$ shown in Figs. 7 and 8 is an average over many samples. The $q(x)$ of each individual aggregate fluctuates around this average. If we define $[\Delta q(x)]^{2}$ as the variance of the $q(x)$, the results for $\Delta q(x)$ also displayed in these figures indicate that it obeys the same scaling law as $q(x)$

$$
\Delta q(x) \simeq G((1-x) /\langle q\rangle) .
$$

This implies that the relative fluctuations remain constant as $N$ increases. ${ }^{13,14}$ In more expressive but approximate terms, this means that the number of branches fluctuates around an increasing average, i.e., branches keep splitting and dying on large scales.

Returning to the spin-glass analogy, isotropic DLA and spin glasses both have a function $q(x)$ which fluctuates from sample to sample. For spin glasses the fluctuations of $q(x)$ are due to the fluctuations of the multivalley landscape, whereas for DLA they represent the random shapes of the clusters. An important difference, however, is that in the case of DLA $\langle q\rangle$ seems to vanish when $N \rightarrow \infty$ and one has to rescale $(1-x)$ to observe nontrivial limits, Eqs. (11) and (13).

\section{Dependence on space dimension}

It is instructive to study the dependence of the scaling function $F(z)$ on space dimension, and to consider a few cases where it may explicitly be calculated analytically. In one dimension accretion occurs on a line, and the aggregate is just a chain of $N$ particles. In the limit $N \rightarrow \infty$, only two values of $q$ are possible: $q=0$ or $q=1$, with 
equal probabilities $\frac{1}{2}$, and there are no sample-to-sample fluctuations (for finite $N$, there are fluctuations due to the random order of arrival of the last particles on each side). The overlap function $q(x)$ is a step function: $q(x)=0$ for $x<\frac{1}{2}, q(x)=1$ for $x>\frac{1}{2}$, so that

$$
\langle q\rangle=\left\langle q_{2}\right\rangle=\frac{1}{2}
$$

and the scaling function $F_{1}(z)$ is simply

$$
F_{1}(x)= \begin{cases}1, & z<1 \\ 0, & z>1\end{cases}
$$

The infinite-dimensional limit corresponds to aggregates grown on a Cayley tree, and it has been shown in previous work ${ }^{18}$ that DLA is then identical to the Eden model, with equal growth probabilities for all boundary sites. It turns out that one can compute the average of $x(q)$ for that system, for any branching ratio $K$ of the Cayley tree. The details of the calculation are given in the Appendix.

The main results are that the average overlap and the second moment of $P(q)$ decrease as

$$
\begin{aligned}
& \langle q\rangle \approx C_{1}(\ln N) / N, \\
& \left\langle q_{2}\right\rangle \approx C_{2} / N,
\end{aligned}
$$

so the exponent defined above in Eq. (4a) is here $\beta_{\infty}=1$. The integrated distribution $x(q)$ obeys a scaling law analogous to Eq. (11), but there is a difference with the finite-dimensional cases: one has to use the second moment $\left\langle q_{2}\right\rangle$ of $P(q)$ rather than $\langle q\rangle$ to obtain the scaling form, as the first moment of the scaling function is infinite. With the variable $y$ defined by

$$
y=(1-x) /\left\langle q_{2}\right\rangle,
$$

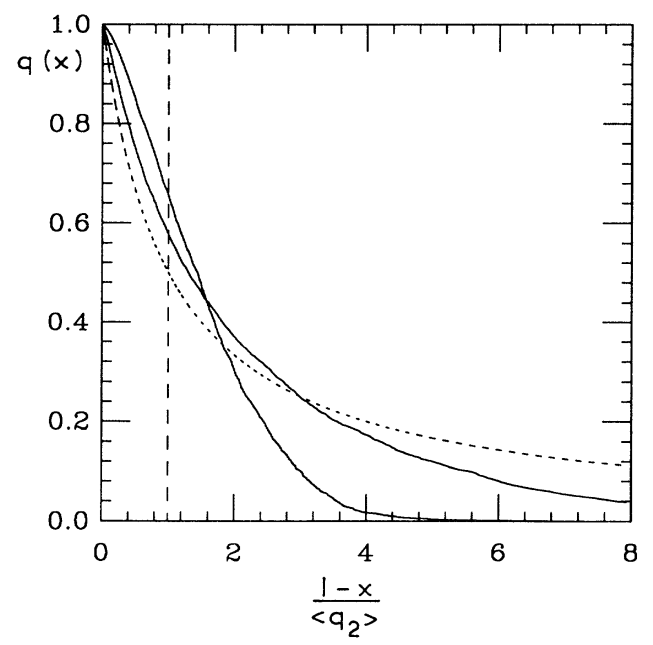

FIG. 9. Overlap function $q(x)$ vs the scaling variable $y=(1-x) /\left\langle q_{2}\right\rangle$, for dimensions $d=1$ (vertical dashed line), $d=2$ and 3, (both solid lines, numerical results on our largest samples), and $d=\infty$ (dotted line, analytical result for the Cayley tree in the limit of infinite branching ratio). the scaling function is given when $K \rightarrow \infty$ by

$$
q=F_{\infty}(y)=1 /(1+y) .
$$

For the tree problem we did not find a way of directly obtaining the analytical expression for the average of $q(x)$, so we calculated it numerically by Monte Carlo sampling, for comparison with the results in $d=2$ and 3. The two averages, over $x$ at fixed $q$ or over $q$ at fixed $x$, seem to coincide asymptotically - but to compute $q(x)$ accurately we had to use a large number of test pairs, $N_{p} \gg N$.

Comparison of the results for various dimensions (Fig. 9) shows clearly a systematic trend, when the space dimension increases, toward a slower decay of the scaling function $F(z)$ for large values of $z$. This is in agreement with the qualitative idea that screening effects are weaker for higher $d$, so the incoming particles can reach deeper into the aggregate, and this gives a higher proportion of overlaps $q$ which are small but nonvanishing in the thermodynamic limit.

\section{ANISOTROPIC AGGREGATES IN $d=2$}

It was initially realized by Ball and $\mathrm{Brady}^{19}$ that the global shape of two-dimensional DLA clusters is very sensitive to anisotropy in the sticking probability; and it is now accepted that anisotropy is a relevant perturbation, in the sense that, for instance, the large-scale behavior of lattice-grown clusters reflects the symmetry of the lattice. It is then natural to investigate how anisotropy affects the overlap distribution defined above. Also, that study provides insight into the relationship between the average overlap $\langle q\rangle$ and the number of branches, for objects where the latter is fixed.

\section{A. Numerical results}

We have studied star-shaped aggregates with $b$-fold symmetry, for $b=2,3$, and 4 , using the antenna method

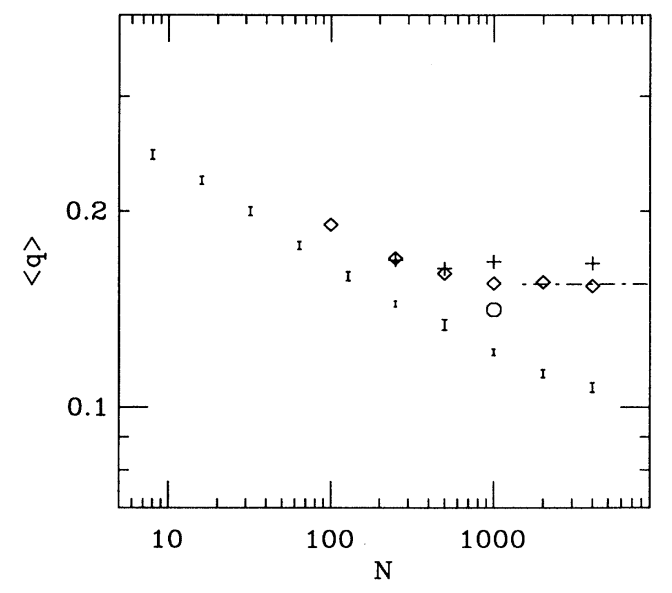

FIG. 10. Average overlap $\langle q\rangle$ vs mass $N$ for 2D star-shaped aggregates of different symmetries: biaxial $(+)$, threefold $(\diamond)$, and fourfold (O). The results for isotropic aggregates are shown for comparison (error bars). The dash-dotted line indicates the analytical result of Sec. IV B for $b=3$. 

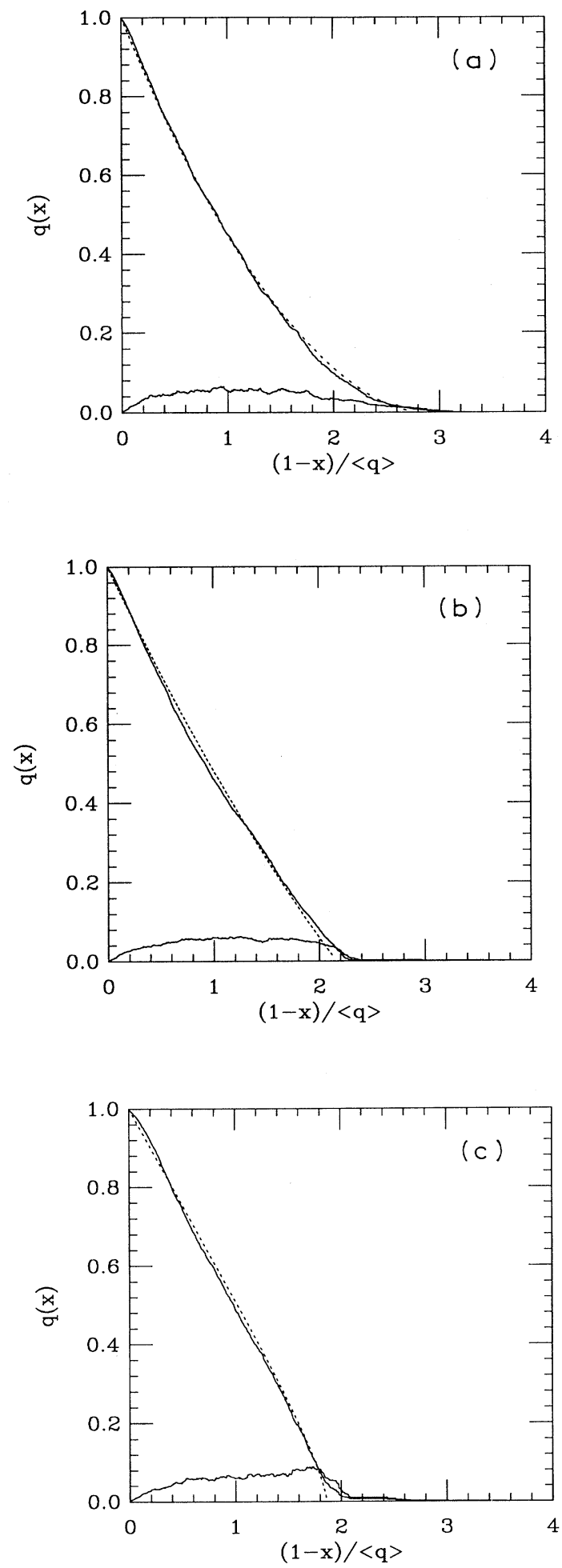

FIG. 11. Overlap function $q(x)$ and fluctuations for anisotropic 2D aggregates with $b$ branches: (a) $b=2, N=4000,50$ samples (horizontal to vertical sticking ratio $\alpha=5$; (b) $b=3$, $N=4000,100$ samples; (c) $b=4, N=1000,50$ samples (noisereduction factor $m=5$ ). The dotted lines correspond to the analytical results for the simplified model studied in Sec. IV B. as described in Sec. II B above, and the noise reduction trick for $b=4$. The first important difference with the isotropic case is that the average overlap has a finite limit for large clusters, which depends on the number $b$ of branches (Fig. 10) and on the noise-reduction parameter, at least for the sizes studied. Moreover, the form of the overlap function $q(x)$ is strongly dependent on the symmetry, with a sharper cutoff for larger $b$, as seen from Fig. 11, where the reduced variable $z=(1-x) /\langle q\rangle$ is used to make comparison with the isotropic case easier.

It is apparent from Figs. 10 and 11 that when the number of branches increases the average overlap decreases and comes closer to the value of $\langle q\rangle$ measured on our largest isotropic clusters, but $F(z)$ becomes less and less similar to the isotropic function $F_{\text {iso }}(z)$ - so that there exists no branch number for which the distribution $P(q)$ approximately fits the isotropic case. For $b=2, F(z)$ and $F_{\text {iso }}(z)$ have some similarity, with a tail for relatively large values of $z$, but the $\langle q\rangle$ are very different. For larger values of $b$, the anisotropic $F(z)$ have a sharp cutoff absent for isotropic DLA.

\section{B. Analytical results on a simplified model}

The difficulty of the DLA model comes from the displacement of the particle-absorbing boundary when accretion takes place. In the presence of a fixed boundary of simple shape, the diffusion problem may be solved in two dimensions, using conformal transformations, ${ }^{9,10}$ and the spatial distribution of the growth probability obtained explicitly. This provides a simplified model of growth if one supposes that no branching occurs and that the cluster shape remains unchanged, up to a scale factor. We now show how to calculate $q(x)$ in that model.

let us consider the growth of a star-shaped cluster with $b$-fold symmetry, the branch length $L$ being supposed to be much larger than its width $W$. The probability $\rho(\lambda)$ that a diffusing particle hits a branch at a distance $d=\lambda L$ from the root is

$$
\rho(\lambda)=\frac{b}{\pi} \frac{\lambda^{b / 2-1}}{\left(1-\lambda^{b}\right)^{1 / 2}}
$$

This expression is given, for instance, as Eq. (2.9) of Ref. 10 , and is obtained by the simple conformal mapping

$$
z \rightarrow f(z)=\left[\left(z^{b / 2}-z^{-b / 2}\right) / 2 i\right]^{2 / b},
$$

which maps the unit circle onto a star with $b$ branches. Since for the case of the unit circle the solution of the Laplace equation is just $\ln (z)$, one obtains for the corresponding solution $V(z)$ of the Laplace equation in the case of the $b$-branch star

$$
\begin{aligned}
V(z) & =\ln \left[f^{-1}(z)\right] \\
& =(2 / b) \ln \left[\left(1-z^{b}\right)^{1 / 2}+i z^{b / 2}\right] .
\end{aligned}
$$

The growth probability is proportional to the normal derivative of $V(z)$, so by differentiating Eq. (21) one recovers Eq. (19), with $\lambda=|z|$.

The distance overlap $q_{d}$ between two test particles is 


$$
q_{d}(A, B)=\min \left(d_{A}, d_{B}\right) / L=\min \left(\lambda_{A}, \lambda_{B}\right)
$$

if they hit the same branch (probability $1 / b$ ), and $q_{d}(A, B)=0$ otherwise. The cumulated distributions of distance overlaps $x_{d}(Q)$ is then given by the probability that $q_{d} \leq Q$ :

$$
x_{d}(Q)=\frac{b-1}{b}+\frac{1}{b}\left[1-\left[\int_{Q}^{1} \rho(\lambda) d \lambda\right]^{2}\right] .
$$

Inserting Eq. (19) into (23), one obtains

$$
x_{d}(Q)=1-\frac{4}{\pi^{2} b}\left[\frac{\pi}{2}-\sin ^{-1}\left(Q^{b / 2}\right)\right]^{2} \text {. }
$$

To make contact with anisotropic DLA clusters and with our original definition of the overlap $q$, which takes into account the "time" of arrival, it is necessary to make a few more physical assumptions. The first common ancestor of two test particles that arrived on the same branch at times $t_{A}$ and $t_{B}$ is close to the oldest particle, i.e.,

$$
q_{A B}=\min \left(t_{A}, t_{B}\right) / N
$$

in other words, their tips are thin and they do not contain long filaments that make a small angle with the branch axis; instead, their structure is more dendritelike. Secondly the time of arrival $t_{k}$ of the $k$ th particle is (on average) directly related to its distance $d_{k}$ to the root through

$$
t_{k} / N \approx\left(d_{k} / L\right)^{\alpha},
$$

with $\alpha=\frac{3}{2}$ in this simplified model. ${ }^{10}$ The time overlap distribution is then given by

$$
x(q)=\int_{0}^{Q=q^{2 / 3}} P_{d}\left(q^{\prime}\right) d q^{\prime}=x_{d}\left(q^{2 / 3}\right)
$$

and the average overlap to be compared to the simulations is

$$
\begin{aligned}
\langle q\rangle & =\int_{0}^{1} Q^{3 / 2} P_{d}(Q) d Q \\
& =\frac{3}{2} \int_{0}^{1} Q^{1 / 2}\left[1-x_{d}(Q)\right] d Q .
\end{aligned}
$$

For the triangular star, one can calculate explicitly this integral

$$
\langle q\rangle=4(\pi-2) /\left(3 \pi^{2}\right)=0.15422 \ldots, \quad b=3
$$

as well as the one giving the mean square overlap

$$
\left\langle q_{2}\right\rangle=\left(\pi^{2}-4\right) /\left(6 \pi^{2}\right)=0.09912 . ., \quad b=3
$$

in close agreement with the numerical results for anisotropic aggregates with threefold symmetry: $\langle q\rangle \approx 0.153 \pm 0.001,\left\langle q_{2}\right\rangle \approx 0.097 \pm 0.001$, for 100 samples of 4000 particles. For the cigar $(b=2)$ and the cross ( $b=4$ ) one obtains, respectively,

$$
\langle q\rangle= \begin{cases}0.18119 \ldots, & b=2 \\ 0.13402 \ldots, & b=4\end{cases}
$$

to be compared with the numerically obtained values $\langle q\rangle=0.166 \pm 0.002$ (50 biaxial aggregates of 4000 parti-

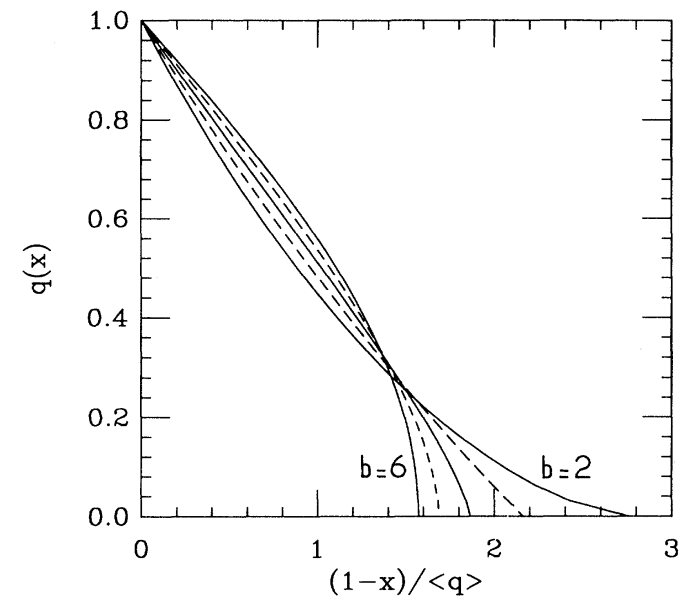

FIG. 12. Analytical results of the overlap function in a simplified model of anisotropic aggregation. The number of branches varies from $b=2$ to $b=6$ (solid lines for even $b$, dashed lines for odd $b$ ).

cles, sticking ratio $\alpha=7)$ and $\langle q\rangle=0.141 \pm 0.002(50$ fourfold symmetric aggregates of 1000 particles, with noise reduction factor $m=5$ ).

When the number $b$ of branches is very large one has

$$
\langle q\rangle_{d} \approx\langle q\rangle \approx b^{-1}+O\left(b^{-2}\right), \quad b>>1
$$

and it is legitimate to expect that for large isotropic clusters $\langle q\rangle^{-1}$ is a quantity that increases like an effective number of branches.

We show $q(x)$, as determined from Eqs. (24) and (27), versus $(1-x) /\langle q\rangle$, for $b$ varying from 2 to 6 , in Fig. 12 . The agreement with the numerical simulations displayed in Figs. (11a)-(11c) is very good, showing that the approximations made in the simplified model are reasonable.

\section{CONCLUSION}

In this paper we have tried to develop an analogy between the random shapes of DLA clusters and the random structure of valleys in the phase space of spin glasses. For DLA clusters we have shown that one can define a function $q(x)$ analogous to the order-parameter function of mean-field spin glasses, and which seems to remain non-self-averaging, even in the limit of infinite clusters. One main difference, however, between DLA and mean-field spin glasses is that as $N$ increases one has to rescale $1-x$ by $\langle q\rangle$ in order to obtain a scaling function $q$ versus $(1-x) /\langle q\rangle$. This rescaling is very reminiscent of a recent proposal by Parisi and Virasoro ${ }^{20}$ to generalize the definition of the broken symmetry of replicas by allowing some rescaling.

The analogy discussed in this paper between a growth model (here DLA) and the spin-glass problem has been already noticed elsewhere: the problems of ballistic deposition or Eden growth are known to be closely related to the problem of directed polymers ${ }^{21,22}$ in a random medi- 
um, which exhibits, at least at the mean-field level, ${ }^{23}$ properties very similar to mean-field spin glasses [broad distribution $P(q)$, non-self-averaging effects]. So one can wonder whether the analogy between growth models and spin glasses is only qualitative or is much deeper, implying quantitative similarities, for example, in the fluctuation laws of $q(x)$.

From the point of view of DLA, our study of the function $q(x)$ seems to distinguish between anisotropic and isotropic clusters. A rough definition of the number of branches as $\langle q\rangle^{-1}$ seems to indicate that the number of branches increases as $N$ increases. This in particular implies that DLA clusters are more complicated than simple self-similar fractals. It would be interesting to understand the $N$ dependence of $\langle q\rangle$ in a finite-dimensional case and to know whether this exponent can be related to the exponents $D_{q}$ of the multifractal structure of the growth sites. ${ }^{24}$

\section{ACKNOWLEDGMENTS}

The Laboratoire de Physique Statistique is associated with Centre National de la Recherche Scientifique and Universities Paris-6 and Paris-7.

\section{APPENDIX: OVERLAPS FOR DLA ON THE CAYLEY TREE}

It has been shown previously ${ }^{18}$ that DLA clusters and Eden clusters have exactly the same statistics on the Cayley tree. This means that screening disappears completely on this particular lattice and that all boundary sites have the same probability to be occupied by the diffusing particle. This property and the fact that the number of boundary sites is completely determined by the total number of particles in the aggregate make the calculation of different statistical properties possible. For example, the mean giration radius and the mean density profile for aggregates of $N$ particles have been computed in Ref. 18 . The Cayley-tree results represents the statistics of aggregates of $N$ particles grown on the Euclidean lattice of Euclidean dimension $d$ tending to infinity $(d \rightarrow \infty)$. Corrections of order $1 / d$ have been computed and discussed in Ref. 25. It appears that the two limits $d \rightarrow \infty$ and $N \rightarrow \infty$ do not commute and that the Bethe-lattice computation $(d \rightarrow \infty$ and then $N \rightarrow \infty)$ does not give a control of the more interesting other limit $(N \rightarrow \infty$ and then $d \rightarrow \infty)$. In spite of this problem, the Cayley-tree DLA is a model that exhibits some of the properties of irreversible growth. It is therefore of some interest to show that the function $P(q)$, as defined in Sec. A, can be computed in this model.

Let us first recall some simple results obtained in Ref. 18. Since there is no screening on the Cayley tree, all growth histories have equal probability. This is why the statistics of DLA clusters is different from the statistics of animals. ${ }^{26}$ Geometrically identical clusters are distinct if they have a different growth history (i.e., if the particles have not been added in the same order) and the weight of a geometrical configuration is proportional to the number of ways it can be grown.

Let us first compute the number $g_{N}$ of different growth

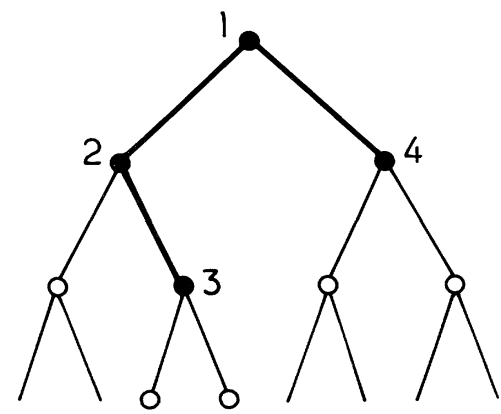

FIG. 13. A cluster of $N=4$ particles (solid circles) numbered in their order of arrival, on a Cayley tree of ramification $K=2$. Diffusing particles can stick on one of the five empty boundary sites (open circles). This particular geometrical structure can be obtained in three distinct ways, among the 24 possible histories of four particles.

histories for clusters of $N$ particles on a Cayley tree of ramification $K$ (Fig. 13). The number of empty boundary sites for a cluster of $j$ particles on this lattice is $1+j(K-1)$. A cluster of $j+1$ particles is created from a cluster of $j$ particles by adding a particle in one of its empty boundary sites. Therefore the number $g_{N}$ of different growth histories is simply

$g_{N}=\prod_{j=0}^{N-1}[1+j(K-1)]=(K-1)^{N} \frac{\Gamma\left(N+\frac{1}{K-1}\right]}{\Gamma\left(\frac{1}{K-1}\right)}$.

(A1)

This is conveniently encoded in the generating function $g(x)$ [denoted by $\widetilde{g}(X)$ in Ref. 17]:

$$
g(X) \equiv \sum_{N=0}^{\infty} \frac{g_{N} X^{N}}{N !}=[1-(K-1) X]^{1 /(1-K)}
$$

It is also helpful to compute the number of growth histories $g_{N, S}$ in which no particle is added on the empty boundary sites that follow the site occupied by the sth particle. This is easily obtained since nothing is changed before the $s$ th particle is added, and at stage $j>S$, among $(K-1)$ empty boundary sites $K$ are not allowed to be filled. The number of available boundary sites at this stage is therefore $(K-1)(j-1)$ and the number $g_{N, S}$ of histories obeying the constraint is

$$
\begin{aligned}
g_{N, S} & =g_{S} \prod_{j=S}^{N-1}(K-1)(j-1) \\
& =g_{S}(K-1)^{N-S} \frac{(N-2) !}{(S-2) !} .
\end{aligned}
$$

This can again be encoded in a generating function 


$$
\begin{aligned}
g(X, S) & \equiv \sum_{N=S}^{\infty} \frac{g(N, S)}{(N-S) !} X^{N} \\
& =g_{S} X^{S}[1-(K-1) X]^{1-S}
\end{aligned}
$$

Having recalled these simple results, we can now proceed to the distribution of overlaps. In the test part a particle pair is thrown. Each particle can land on any of the $1+N(K-1)$ boundary sites with equal probability. So for a particular aggregate each one of the $[1+N(K-1)]^{2}$ ordered pairs of boundary sites can be chosen with equal probability. For each pair, there is a particle in the aggregate that is the last common ancestor of the two boundary sites. If this particle is the $S$ th one in the aggregate the overlap of the couple is defined by $q=S / N$. The suitably normalized histogram of values of $q$ is the function $P_{a}(q)$ for the aggregate $a$. Let us denote by $C_{a}(N, S)$ the number of couples that have the $S$ th particle as last common ancestor. Then

$$
\sum_{S=1}^{N} C_{a}(N, h)=[1+N(K-1)]^{2}
$$

and

$$
P_{a}(q)=\frac{C_{a}(N, S)}{[1+N(K-1)]^{2}} \quad \text { where } S=q N .
$$

We are going to compute the mean value $\langle P(q)\rangle$ of $P_{a}(q)$ over all aggregates:

$$
\begin{aligned}
\langle P(q)\rangle & \equiv \frac{1}{g_{N}} \sum_{a} P_{a}(q) \\
& =\frac{1}{g_{N}[1+N(K-1)]^{2}} C(N, q N),
\end{aligned}
$$

where we have defined the total number of couples (over all aggregates) that have overlap $S$

$$
C(N, S)=\sum_{a} C_{a}(N, S)
$$

This last quantity can now be computed by counting the aggregates of $N$ particles in a particular way. We first group together the aggregates that have the same number $n_{1}, \ldots, n_{K}$ of particles on the $K$ branches following the $S$ th particles. The number of such clusters is

$$
\begin{aligned}
& g_{n_{1}} \cdots g_{n_{K}} \cdots g_{m, S} \frac{(N-S) !}{(m-S) ! n_{1} ! \cdots n_{K} !} \\
& \text { where } m=N-\left(n_{1}+\cdots+n_{K}\right)
\end{aligned}
$$

since after the addition of the $S$ th particle $N-S$ particles remain to be added. If $n_{1}, \ldots, n_{K}$ particles are added on the $K$ branches following the $S$ th particle, $m-S$ particles remain to be added elsewhere. Therefore the aggregate can be viewed as a cluster of $m$ particles with no particle following the $S$ th one, plus $K$ clusters of $n_{1}, \ldots, n_{K}$ particles rooted at the $S$ th particle. The combinatorial factor appears, since after time $S$, at each time step any of the $K+1$ clusters (the main cluster and the $K$ clusters rooted at the particle) can grow.

For each such aggregate a particle pair has an overlap $s$ because of either of the following.

(i) The two particles fall on two different branches following the $S$ th particle and there are

$$
\sum_{i \neq j}\left[1+n_{i}(K-1)\right]\left[1+n_{j}(K-1)\right]
$$

such possibilities.

(ii) One of the branches following the Sth particle is empty and the two particles fall on the corresponding boundary site. There are obviously $\sum_{i} \delta_{n_{i}, 0}$ such possibilities.

Therefore the contribution to $C(N, S)$ of these clusters is

$$
g_{n_{1}} \cdots g_{n_{K}} \cdots g_{m, S} \frac{(N-S) !}{(m-S) ! n_{1} ! \cdots n_{K} !}\left(\sum_{i \neq j}\left[1+n_{i}(K-1)\right]\left[1+n_{k}(K-1)\right]+\sum_{i} \delta_{n_{i}, 0}\right) .
$$

The total number of pairs is obtained by a summation over the different groups of aggregates that is over the $\left\{n_{i}\right\}$. troducing the generating function

$$
C(X) \equiv \sum_{N=S}^{\infty} \frac{C(N, S)}{(N-S) !} X^{N}
$$

one obtains, using (A10),

$$
\begin{aligned}
C(X) & =g(X, S) g^{K-2}(X)\left\{K(K-1)\left[g(X)+(K-1) X g^{\prime}(X)\right]^{2}+K g(X)\right\} \\
& =g_{S} X^{S}[1-(K-1) X]^{-S}\left[K(K-1)[1-(K-1) X] \frac{2 K-1}{1-K}+K\right] .
\end{aligned}
$$

Therefore the total number of couples of overlap $S$ is

$$
C(N, S)=g_{s}(K-1)^{N-2}\left(K(K-1) \frac{\Gamma\left[N+\frac{2 K-1}{K-1}\right)}{\Gamma\left(S+\frac{2 K-1}{K-1}\right)}+K \frac{(N-1) !}{(S-1) !}\right) \text {. }
$$


The function $\langle P(q)\rangle$ is obtained by normalizing $C(N, S)$ using (A7) and (A 1), and the ensuing formula is the main result of this appendix:

$$
\left.\langle P(q)\rangle=\frac{K}{[1+N(K-1)]^{2}}(K-1) \frac{\left(N+\frac{1}{K-1}\right]\left[N+\frac{K}{K-1}\right)}{\left[S+\frac{1}{K-1}\right]\left[S+\frac{K}{K-1}\right]}+\frac{\Gamma\left(S+\frac{1}{K-1}\right)}{\Gamma\left[N+\frac{1}{K-1}\right]} \frac{(N-1) !}{(S-1) !}\right]_{S=q N} .
$$

The first term represents the contribution from pairs of particles falling on different boundary sites. The second one comes from pairs of particles that fall on the same site, and its contribution is smaller by $1 / K$ than the first term in the large- $K$ limit. We neglect it in the following for simplicity. The results are nonetheless exact for any $K$ if during the test part one keeps only the pairs of test particles that have fallen on different sites and modifies the normalizing constant accordingly. So we replace formula (A14) by

$$
\begin{aligned}
\langle P(q)\rangle & \left.=\frac{K}{[1+N(K-1)] N(K-1)} \mid(K-1) \frac{\left(N+\frac{1}{K-1}\right)\left(N+\frac{K}{K-1}\right)}{\left(S+\frac{1}{K-1}\right)\left(S+\frac{K}{K-1}\right)}\right] \\
& =\left.\frac{K}{N} \frac{[N(K-1)+K]}{[S(K-1)+1][S(K-1)+K]}\right|_{s=q N} .
\end{aligned}
$$

The moments of $\langle P(q)\rangle$ can be easily computed. For instance,

$$
\begin{aligned}
& \langle q\rangle=\frac{1}{N} \sum_{S=1}^{N} S\left\langle P\left(\frac{S}{N}\right)\right\rangle \sim \frac{K}{K-1} \frac{\ln N}{N}, \\
& \left\langle q^{2}\right\rangle=\frac{1}{N^{2}} \sum_{S=1}^{N} S^{2}\left\langle P\left(\frac{S}{N}\right)\right\rangle \sim \frac{K}{K-1} \frac{1}{N}, \\
& \left\langle q^{i}\right\rangle=\frac{1}{N^{2}} \sum_{S=1}^{N} S^{i}\left\langle P\left(\frac{S}{N}\right)\right\rangle \sim \frac{K}{K-1} \frac{1}{i-1} \frac{1}{N} .
\end{aligned}
$$

The function $X(q)$ is given by

$$
X(q)=\sum_{1 \leq S \leq N q}\langle P(S, N)\rangle=q \frac{\frac{K}{N}+(K-1)}{\frac{K}{N}+(K-1) q} .
$$

For DLA in two and three dimensions, we have argued that if $1-X(q)$ is normalized by a moment of $P(q)$, one obtains a well-defined nontrivial function in the limit $N \rightarrow \infty$. This is also true on the Cayley tree (except that one should not take the first moment that has a logarithmically anomalous scaling behavior). For example,

$$
\frac{1-X(q)}{\left\langle q^{2}\right\rangle}=\frac{(K-1)(1-q)}{\frac{K}{N}+(K-1) q},
$$

and in the limit $N \rightarrow \infty$,

$$
\frac{1-X(q)}{\left\langle q^{2}\right\rangle}=\frac{1}{9}-1
$$

${ }^{1}$ T. Witten and L. Sander, Phys. Rev. Lett. 47, 1400 (1981).

${ }^{2}$ Recent general introductions to growth models are given by $\mathrm{T}$. Vicsek, Fractal Growth Phenomenon (World Scientific, Singapore, 1989); J. Feder, Fractals (Plenum, New York, 1988).

${ }^{3} \mathrm{P}$. Meakin, in Phase Transitions and Critical Phenomena, edited by C. Domb and J. Lebowitz (Academic, New York, 1988), Vol. 12, pp. 330-489.

${ }^{4}$ F. Argoul, A. Arnéodo, J. Elezgaray, G. Grasseau, and R. Murenzi, Phys. Lett. A 135, 327 (1989).

5J. Vannimenus and X. G. Viennot, J. Stat. Phys. 54, 1529 (1989); E. L. Hinrichsen, K. J. Maloy, J. Feder, and T. Jossang, J. Phys. A 22, L271 (1989).

${ }^{6}$ B. B. Mandelbrot and T. Vicsek, J. Phys. A 22, L377 (1989).

${ }^{7}$ P. Alstrom, P. A. Trunfio, and H. E. Stanley, Phys. Rev. A 41, 3403 (1990).

${ }^{8}$ A. Arnéodo, Y. Couder, G. Grasseau, V. Hakim, and M. Ra- baud, Phys. Rev. Lett. 63, 984 (1989).

${ }^{9}$ F. Family and H. G. E. Hentschel, Faraday Disc. Chem. Soc. 83, 139 (1987); K. Kassner and F. Family, Phys. Rev. A 39, 4797 (1989).

${ }^{10}$ J.-P. Eckmann, P. Meakin, I. Procaccia, and R. Zeitak, Phys. Rev. A 39, 3185 (1989).

${ }^{11}$ See, for instance, P. Meakin, in Phase Transitions and Critical Phenomena, Ref. 3, pp. 405-410.

${ }^{12}$ Alternatively, one could solve the Laplace equation with the proper boundary conditions on the cluster and obtain the growth probabilities on every growth site, as done by L. A. Turkevitch and H. Scher [Phys. Rev. Lett. 55, 1026 (1985)]; then use them as weights for the overlaps $q_{A B}$ computed for all pairs of growth sites. That procedure would be more accurate in principle but much more demanding in computer time. 
${ }^{13}$ M. Mézard, G. Parisi, and M. Virasoro Spin Glass Theory and Beyond (World Scientific, Singapore, 1987).

${ }^{14}$ A. J. Bray, M. A. Moore, and A. P. Young, J. Phys. C 17, L149 (1984).

${ }^{15}$ R. C. Ball, Physica 104A, 62 (1986); and P. Meakin, in Phase Transitions and Critical Phenomena (Ref. 3), pp. 377-380.

16J. Nittmann and H. E. Stanley, Nature 321, 663 (1986); and P. Meakin, in Phase Transitions and Critical Phenomena (Ref. 3), p. 374.

${ }^{17} \mathrm{H}$. Herrmann (private communication).

${ }^{18}$ J. Vannimenus, B. Nickel, and V. Hakim, Phys. Rev. B 30, 391 (1984).
${ }^{19}$ R. C. Ball and R. M. Brady, J. Phys. A 18, L809 (1985).

${ }^{20}$ G. Parisi and M. A. Virasoro, J. Phys. (Paris) 50, 3317 (1989).

${ }^{21}$ M. Kardar, G. Parisi, and Y. Zhang, Phys. Rev. Lett. 56, 889 (1986).

22J. Krug and H. Spohn (unpublished).

${ }^{23}$ B. Derrida and H. Spohn, J. Stat. Phys. 51, 817 (1988).

${ }^{24}$ A. Coniglio and M. Zannetti, Physica D 38, 37 (1989).

${ }^{25}$ G. Parisi and Y. Zhang, Phys. Rev. Lett. 53, 1791 (1984); D. Dhar, ibid. 54, 2058 (1985); R. Friedberg, Ann. Phys. 171, 321 (1986); W. M. Zheng, Phys. Rev. A 35, 4904 (1987); 36, 5851 (1987).

${ }^{26}$ M. E. Fischer and J. W. Essam, J. Math. Phys. 2, 609 (1961). 